\title{
Evolution of COVID-19 Pulmonary Fibrosis-Like Residual Changes Over Time - Longitudinal Chest CT up to 9 Months After Disease Onset: a Single-Center Case Series
}

\author{
Antonio Poerio ${ }^{1}$ (D) Eleonora Carlicchi ${ }^{2} \cdot$ Ludovica Lotrecchiano $^{3}$. Chiara Praticò ${ }^{4}$. Giacomo Mistè ${ }^{5}$. \\ Saverio Scavello ${ }^{5}$ Miria Morsiani ${ }^{1} \cdot$ Maurizio Zompatori $^{6} \cdot$ Rodolfo Ferrari $^{4}$
}

Accepted: 8 February 2022 / Published online: 14 February 2022

(c) The Author(s), under exclusive licence to Springer Nature Switzerland AG 2022

\begin{abstract}
The aim of the study was to evaluate the temporal evolution of fibrotic-like pulmonary interstitial abnormalities secondary to Sars-CoV-2 virus (COVID-19) pneumonia detected on chest-CTs of patients hospitalized for COVID-19 infection. We retrospectively reviewed chest-CTs obtained up to 9 months after disease onset in a group of patients with COVID-19 pneumonia and CT features suggestive of lung fibrosis at the first follow-up after hospital discharge. We observed a complete and unexpected resolution of all interstitial abnormalities, including reticulations and bronchial dilatation, in a period of about 6-9 months after discharge. Interstitial fibrotic-like changes detectable in the first months after COVID-19 pneumonia could be slowly or very slowly resolving but still completely reversible and probably secondary to an organizing pneumonia reaction.
\end{abstract}

Keywords COVID-19 $\cdot$ Diffuse lung disease $\cdot$ Organizing pneumonia $\cdot$ Pulmonary fibrosis $\cdot$ HRCT

\section{Introduction}

Since its appearance at the end of 2019, the Sars-CoV-2 virus (COVID-19) has caused almost 5 million deaths and more than 245 million confirmed cases of infection [1].

This article is part of the Topical Collection on COVID-19

Antonio Poerio
a.poerio@ausl.imola.bo.it
Eleonora Carlicchi
eleonora.carlicchi@gmail.com
Ludovica Lotrecchiano
ludovica.2611@gmail.com
Chiara Praticò
c.pratico@ausl.imola.bo.it
Giacomo Mistè
g.miste@ausl.imola.bo.it
Saverio Scavello
s.scavello@ausl.imola.bo.it
Miria Morsiani
m.morsiani@ausl.imola.bo.it
Maurizio Zompatori
maurizio.zompatori@unibo.it

Therefore, the possibility that even just a percentage of patients may develop pulmonary long-term sequelae, such as pulmonary fibrosis, represents a serious concern for healthcare services worldwide [2].

Although the importance of follow-up for assessing long-term pulmonary sequelae has been highlighted [3-5], sparse data about the medium and long-term evolution are

Rodolfo Ferrari

r.ferrari@ausl.imola.bo.it

1 Radiology Unit, S. Maria Della Scaletta Hospital, via Montericco 4, 40026 Imola, BO, Italy

2 Postgraduate School in Radiodiagnostics, Università Degli Studi Di Milano, Milan, Italy

3 Department of Radiology, IRCCS Ospedale San Raffaele Turro, via Stamira d'Ancona 20, 20127 Milan, Italy

4 Emergency Care Unit, S. Maria Della Scaletta Hospital, via Montericco 4, 40026 Imola, BO, Italy

5 Internal Medicine Unit, S. Maria Della Scaletta Hospital, via Montericco 4, 40026 Imola, BO, Italy

6 Department of Radiology, Multimedica IRCCS, San Giuseppe Hospital, Milano, Italy 
available, with most reports exploring a 3-month follow-up period, few exploring a 6-month follow-up and only one study reporting a 1-year follow-up after discharge [6-11].

In this series, we evaluated the radiological evolution of interstitial lung alterations suggestive of pulmonary fibrosis detectable in patients treated and discharged for COVID-19 pneumonia through longitudinal chest CT scans obtained up to 9 months after discharge, one of the longest follow-up reported in literature for this class of patients so far. Furthermore, to the best of our knowledge, this is the first work in which only patients presenting a CT fibrosis-like pattern were selected, in order to analyze in detail its temporal evolution and evaluate its potential reversibility.

\section{Materials and Methods}

The study was approved by the "Area Vasta Emilia Centro" Independent Ethical Committee (CE-AVEC; study ID: EM335-2021-20111-EM1-OSS-AUSLIM) and was conducted in compliance with the Declaration of Helsinki. All images were acquired as a standard of care.

We retrospectively selected symptomatic patients admitted to the Emergency Department of our Institution between March 8 and April 3, 2020, who met the following criteria: (1) CT evidence of COVID-19 pneumonia, subsequently confirmed by RT-PCR positivity for SARS-Cov-2; (2) signs of pulmonary architectural distortion suggestive of pulmonary fibrosis at the first CT follow-up performed after discharge; and (3) presence of at least a second CT follow-up in the following months. When present, a third CT follow-up was also evaluated.

We performed a retrospective analysis of patients' electronic medical records evaluating clinical, imaging, and laboratory data. We analyzed patients' age, arterial blood gas analysis parameters $\left(\mathrm{PaO}_{2}\right.$ and $\left.\mathrm{PaO}_{2} / \mathrm{FiO}_{2}\right)$, the length of hospitalization and any episode of rapid worsening of respiratory function with the need for intensive or sub-intensive assistance, and its duration. We also assessed how many patients needed $\mathrm{O}_{2}$-therapy, and invasive or non-invasive mechanical ventilation (NIV and CPAP) and its duration.

After discharge, patients underwent respiratory followup, consisting of pulmonary function tests (PFTs) including forced expiratory volume in $1 \mathrm{~s}$ (FEV1), forced vital capacity (FVC), FEV1/FVC ratio, total lung capacity (TLC), and lung diffusing capacity for carbon monoxide (DLCO) and DLCO divided by alveolar volume (Krogh index - KCO) measured in a single breath test. When available, respiratory followup data were included in the analysis, compared with predicted normal values (based on the patient's height, age, sex, and race) and reported as percentages of predicted values according to the recommendations of the American Thoracic Society (ATS) and the European Respiratory Society (ERS).

\section{CT Protocol}

All CTs were obtained using a 16-slice multidetector row CT scanner (Brilliance iCT SP, Philips) without ECGsynchronization, with the patient in the supine position and during a deep inspiratory breath-hold. Images were acquired with the use of high-resolution tube voltage and current settings $(120 \mathrm{kV}, 200 \mathrm{mAs}$, varying depending on patients' body mass index, $16 \times 0.75$ collimation and pitch $=0.938$ ) with a scanning range covering the area from lungs apices to the diaphragm, generally with a cranio-caudal scanning direction. Images were reconstructed using a high-spatial-frequency kernel algorithm with a $1.00-\mathrm{mm}$ slice thickness, and viewed at window settings levels appropriate for the examination of lung parenchyma (window level, -600 to $-700 \mathrm{HU}$; window width, 1200-1500 HU).

\section{Review of CT Images}

All CTs were evaluated in consensus by three radiologists, respectively, with 5, 10, and more than 30 years of experience in thoracic imaging. Definitions of CT findings described in this study were based on the standard glossary for thoracic imaging reported by the Fleischner Society [12].

At baseline CT, we evaluated the classical signs of COVID-19 pneumonia: ground-glass opacities (GGo), crazy-paving and consolidations [13, 14], and quantified the extension of parenchymal disease using the CT-visual score method (VS). The VS is a semi-quantitative method largely used to assess the parenchymal volume affected by disease on CT images; it is obtained by dividing each lung into 3 pre-established zones: the upper zone defined as above the aortic arch, the middle zone defined as between the aortic arch and pulmonary veins, and the lower zone defined as at or below the pulmonary veins. The overall extent of lung abnormalities is evaluated for each zone and for each side on axial images using a 5-point scale giving a score from 0 to 4 ( 0 : no involvement; $1: 1-25 \%$ involvement; $2: 26-50 \%$; 3: $51-75 \%$; and $4: 76-100 \%)$. The final score is expressed as a percentage of total lung volume $[15,16]$.

At the first follow-up, we considered the following signs as indicative of pulmonary architectural distortion and suggestive of pulmonary fibrosis: irregular interface, reticular opacities, parenchymal bands, and bronchial dilatation [17, 18]; axial and coronal distribution were also assessed and compared with baseline CT. Furthermore, we examined the presence of more peculiar signs such as perilobular pattern (represented by arcadelike/polygonal opacities, mostly with subpleural distribution) [19], interlobular septal thickening, halo sign, and reversed halo sign [14, 20]. 
In subsequent follow-up CTs, we evaluated the evolution of interstitial changes found in former scans.

\section{Results}

Between March 8 and April 3, 2020, 192 patients admitted to the Emergency Department with respiratory symptoms underwent a chest $\mathrm{CT}$, considered to be the baseline $\mathrm{CT}$, and were subsequently diagnosed with COVID-19 infection by RT-PCR positivity for SARS-Cov- 2 .

In total, 22 out of 192 patients had a first CT followup in the first 2 months after discharge (median duration 34 days; range 29-55 days) since some had had a severe form of pneumonia, with prolonged hospitalization and complex clinical course, and others had less severe disease, with CT performed at the request of the referring physician in relation to persistent residual dyspnea. Nine of the $22(40 \%)$ patients showed persisting lung interstitial anomalies at the first CT follow-up; of these 9 patients, 8 had a 3-month CT follow-up (median duration 111 days; range 80-146 days) and 5 had a further CT follow-up at 8-9 months (median duration 237 days; range 186-272 days) and were therefore included in the analysis.

Of the 13 patients who did not show persistent signs of interstitial disease at the first $\mathrm{CT}$ follow-up performed after discharge, 7 had a baseline CT presentation pattern characterized by bilateral GGo and consolidations with prevalent peripheral distribution and a mean VS of 30\%, 4 had mild forms of interstitial pneumonia and were discharged after two days (VS 10\%), and 2 were admitted to intensive care unit with severe pneumonia with diffuse alveolar damage.

The patients' mean age was $71 \pm 10.5$ years; initial mean $\mathrm{PaO}_{2} / \mathrm{FiO}_{2}$ ratio was $277.1 \pm 80.3$ with an average parenchymal involvement of $28.75 \% \pm 21 \%$ assessed by CT VS.

The median length of hospitalization was 29 days (range 19-32 days), during which all patients (8/8) received oxygen-therapy, five patients needed transfer to intensive and sub-intensive care units for a median duration of 11 days (range 5-17 days), four patients received non-invasive mechanical ventilation, and one patient required invasive mechanical ventilation (for 10 days).

Four patients experienced a rapid clinical deterioration during hospitalization period: one after extubation, with a $\mathrm{PaO}_{2} / \mathrm{FiO}_{2}$ ratio of 150 treated with NIV; two patients during low-flow $\mathrm{O}_{2}$-therapy (one of them with a $\mathrm{PaO} 2 /$ $\mathrm{FiO} 2$ ratio of 124 treated with $\mathrm{O}_{2}$-therapy with $15 \mathrm{l} / \mathrm{min}$ reservoir and the other one with a $\mathrm{PaO}_{2} / \mathrm{FiO}_{2}$ ratio of 125 and treated with CPAP); one patient during high-flow $\mathrm{O}_{2}$-therapy, with a $\mathrm{PaO}_{2} / \mathrm{FiO}_{2}$ ratio of 76 , treated with CPAP.

\section{Baseline CT Scan}

Upon admission, one of the eight patients presented with diffuse, bilateral areas of parenchymal consolidation and GGo, predominant in peripheral lung regions, with $80 \%$ lung volume involvement quantified by VS; another patient had nodular GGo with bilateral, patchy, and peripheral distribution, and a disease extent of 15-20\% of lung volume quantified by VS; three others presented bilateral homogenous GGo with a VS of 30-35\%; two patients showed extensive GGo with crazypaving in the upper lobes and consolidations in the lower lobes with a VS of 35\%; and one patient presented widespread GGo with crazy-paving in the upper and lower lobes with a VS of $20 \%$. Two patients also had evidence of perilobular pattern.

\section{First CT Follow-Up}

The 1st CT follow-up was performed during the first 2 months after discharge (median delay 34 days; range 29-55 days). We observed a significant reduction in extent and density of GGo in all cases, but a new irregular interface was always found. Six of the eight patients developed reticular opacities, four of them with a real "coarse reticular pattern"; five patients showed parenchymal bands; and one patient had coarse and irregular consolidations (Fig. 1).

All alterations had peripheral distribution on the axial plane, while no prevalent distribution was found on the coronal plane. In all cases, a clear and characteristic subpleural sparing was observed. Five of the eight patients showed a perilobular pattern (Fig. 2); six patients had bronchial and bronchiolar distortion inside or close to reticulations and parenchymal bands. In one patient, parenchymal abnormalities also involved areas free from disease in the baseline CT, with complete resorption of the former lesions, suggesting a migratory behavior.

\section{Second CT Follow-Up}

The second CT follow-up was performed about 3-4 months after discharge (median delay 111 days; range $80-146$ days). We found an almost complete resolution of parenchymal distortion abnormalities, with complete resorption of reticulations and parenchymal bands, and complete regression of associated bronchiectasis (Fig. 3).

In three patients, we observed a thin curvilinear subpleural line, while barely perceptible GGo areas were still observable in six of them.

\section{Third CT Follow-Up}

Five of the eight patients underwent a 3rd CT follow-up about 8-9 months after discharge (median delay 237 days; range 186-272 days). 
Fig. 1 a-d HRCT images of different patients with COVID-19 pneumonia showing typical imaging features indicative of fibrosis: irregular interface (a), sub-pleural bandlike parenchymal consolidations (black arrows in b), coarse reticular opacities (white circle in c) and bronchial dilatation, observed both on axial image (white arrows in c) and on coronal reconstruction (white arrow in d)

Fig. 2 a-d Axial HRCT images of different patients with COVID-19 pneumonia showing irregular, poorly defined arcadelike or polygonal opacities with subpleural distribution (black circles in $\mathbf{a}$ and $\mathbf{c}$ ), also referred to as perilobular pattern, better seen in the enlarged details (b and d)
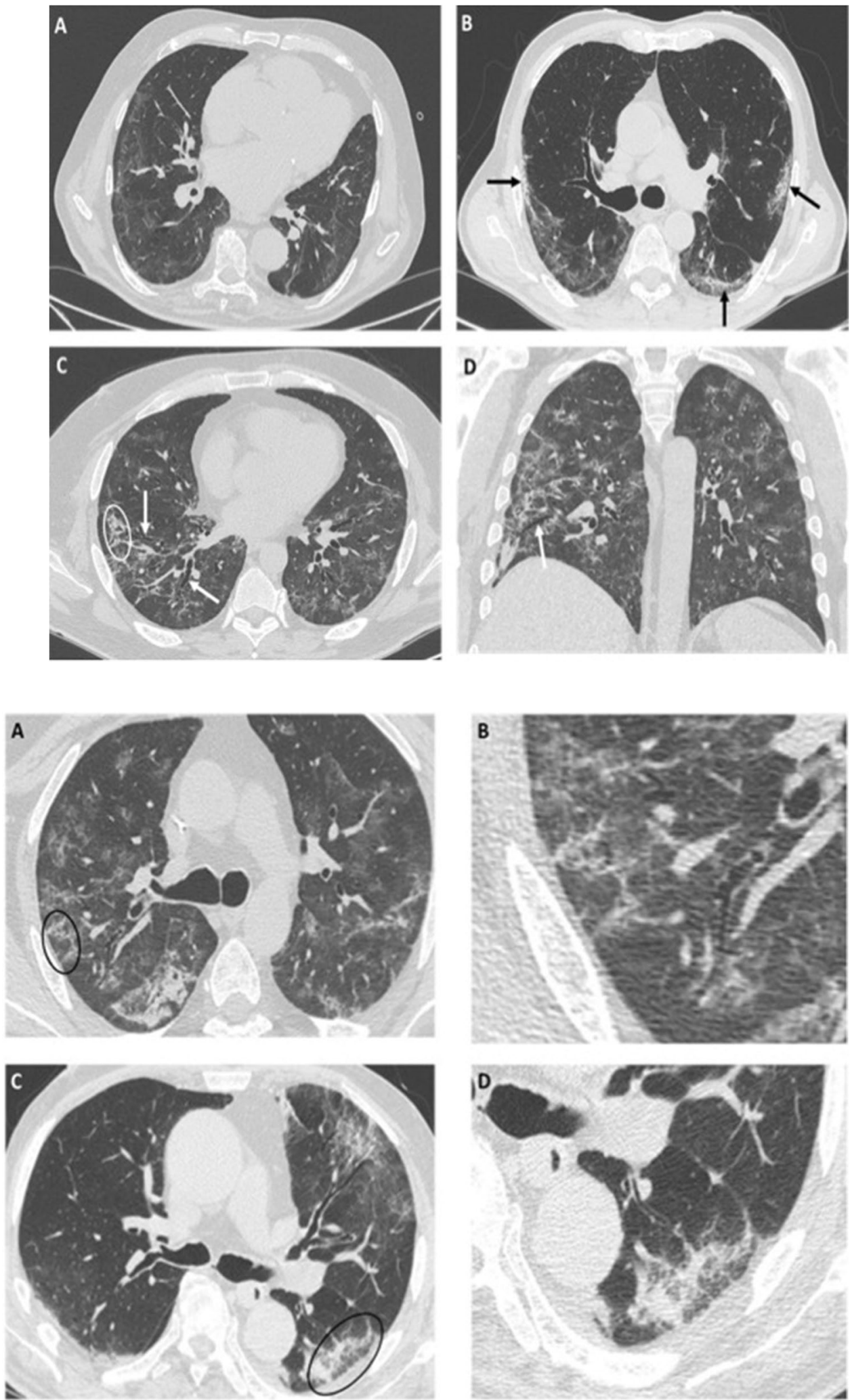

In all patients, we observed a complete regression of residual parenchymal alterations: in one case, the curvilinear subpleural line was no longer visible, while we observed a complete resorption of the already minimum GGo in four patients (Figs. 4, 5, and 6). 
Fig. 3 Enlarged detail of HRCT axial (a-b) and coronal (c-d) images, of two patients with COVID-19 pneumonia showing reticular peripheral opacities associated with bronchial dilatation (white arrows in $\mathbf{a}$ and $\mathbf{c}$ ), which completely resolve at the follow-up scan performed 2 months later (b and $\mathbf{d}$, respectively)
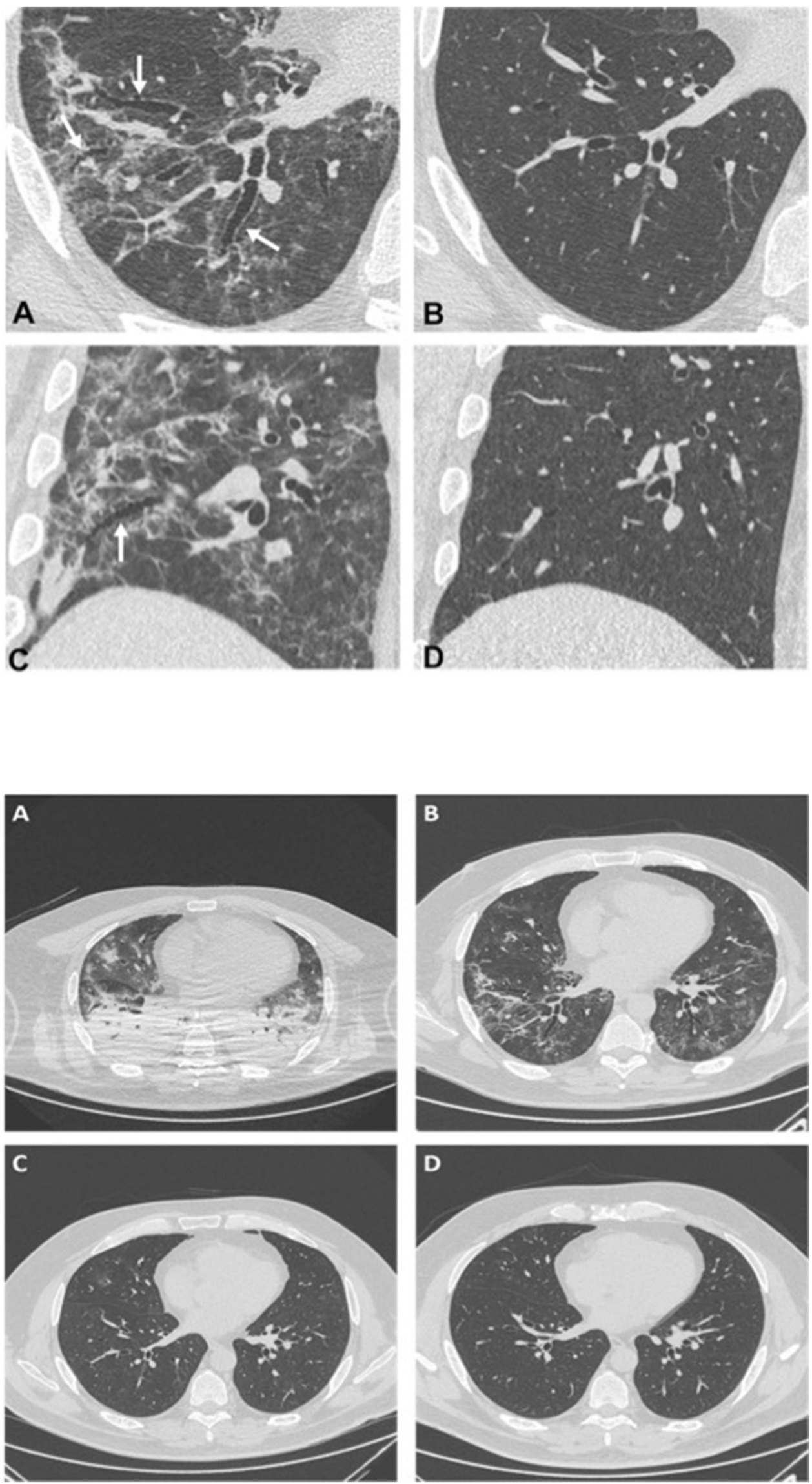

SN Comprehensive Clinical Medicine A SPRINGER NATURE journal 
Fig. 5 Coronal HRCT images of a 76-year-old man with COVID-19 pneumonia, depicting the posterior lung regions at different examination times: baseline scan (a), 1-month follow-up (b), 3-month followup (c), and 8-month follow-up (d). The baseline scan (a) shows bilateral ground-glass opacities, with posterior and peripheral prevalent distribution, more evident in the right lung. In the 1-month follow-up scan (b), ground-glass areas are replaced by bilateral, sub-pleural reticular opacities, mainly seen in the posterior and peripheral regions, with associated bronchiectasis visible in the right lung base (white arrows in b) suggesting fibrosis. The 3-month followup scan shows a regression of parenchymal abnormalities, with bilateral ground-glass opacities posteriorly and peripherally distributed and complete resolution at the 8 months scan follow-up (d)

Fig. 6 Axial HRCT images of a 82-year-old man with COVID-19 pneumonia, at the same level, acquired at different times: baseline scan (a), 1-month follow-up (b), 3-month follow-up (c), and 6-month follow-up (d). In the baseline scan (a), a ground-glass opacity is seen in the left lower lobe (white arrow in a); the 1-month scan shows an increased extension of ground-glass opacities, now also observed in the right lung, with more dense reticular opacities, mainly seen in the left lower lobe with sub-pleural distribution, suggestive for pulmonary fibrosis. Parenchymal abnormalities improve at the 3-month follow-up (c) with only tiny ground-glass opacities, principally observed in the left lower lobe, and completely resolve at the 6-month followup (d)
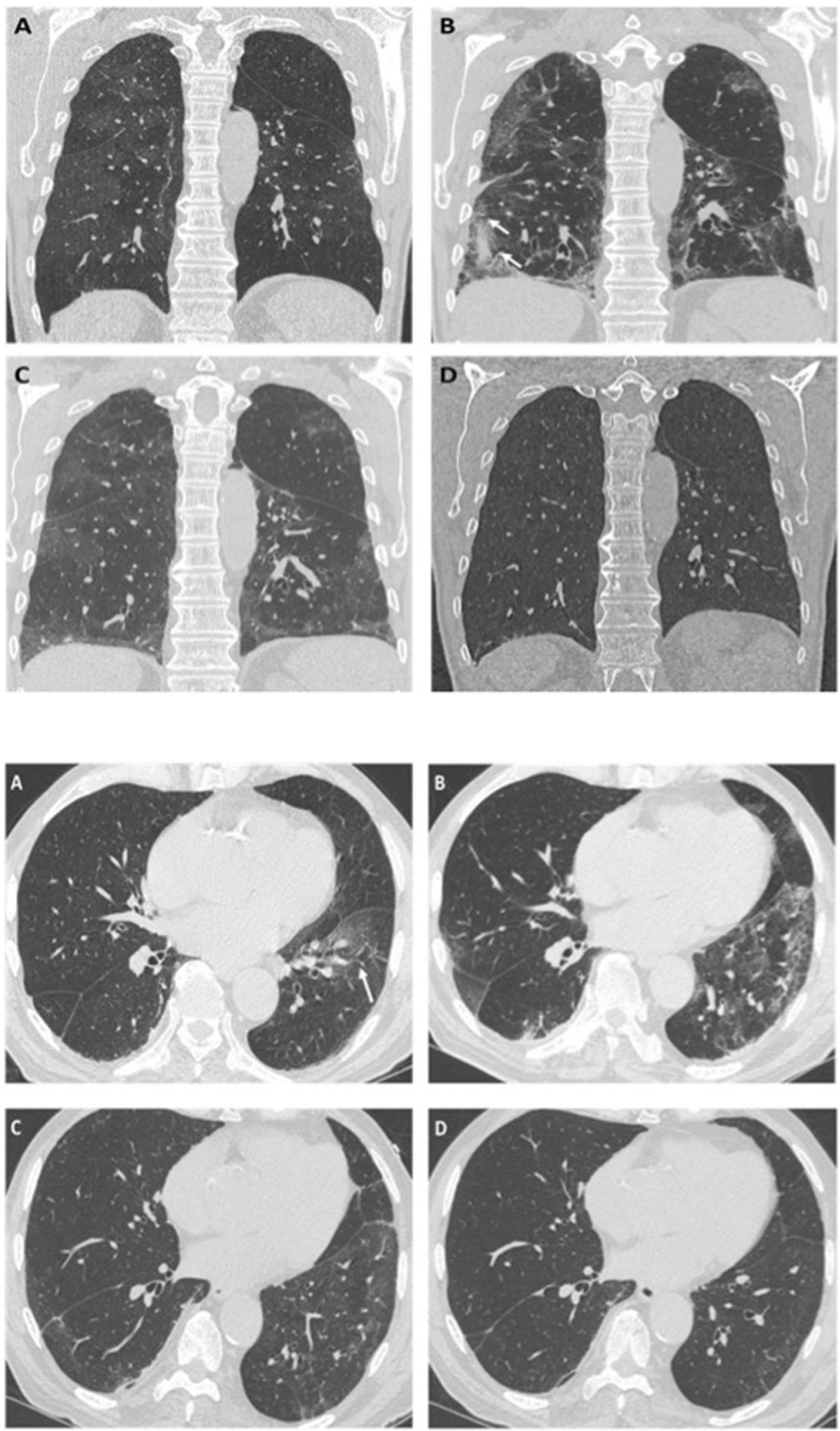


\section{Functional Respiratory Follow-Up}

Respiratory follow-up by PFTs was performed approximately 5 months after discharge (median 158 days; range 140-184 days).

Two of the 8 patients, with already known COPD as a comorbidity, showed obstructive deficits at spirometry, one mild and one moderate, but these results were unchanged from the spirometry performed before COVID19 pneumonia.

One of the 8 patients showed moderate restrictive deficit but no reduction in DLCO. Five patients showed normal lung function at spirometry, without significant deficits.

Five of the 8 patients had a DLCO reduction, of which 3 were mild $(\geq 70 \%)$ and without $\mathrm{KCO}$ changes $(\geq 80 \%)$ and 2 were moderate $(<60 \%)$ with a concurrent $\mathrm{KCO}$ reduction ( $<80 \%$ and $<60 \%$, respectively); however, these patients had a known history of COPD and both DLCO and KCO values were essentially unchanged to those obtained before COVID-19 pneumonia.

\section{Discussion}

The risk of developing long-term pulmonary sequelae after COVID-19 pneumonia is still a matter of debate. Furthermore, only few works have assessed in detail the medium to long-term radiological evolution of the interstitial changes secondary to this infection, and the longest follow-up study available spans 1 year [6-11].

In one of the very first studies on the subject, $\mathrm{Yu}$ and colleagues analyzed the follow-up CTs of 32 patients discharged after COVID-19 pneumonia and found signs of pulmonary architectural distortion in 14 patients (44\%) [7]. In another work, Wei and colleagues analyzed the follow-up CTs of 59 patients with previous COVID-19 pneumonia, 23 of which (39\%) showed signs of parenchymal distortion [8]. In both works, the authors assessed the presence of parenchymal bands, reticulations, and bronchial distortion, as we did, but the only follow-up CT was performed less than one month after disease onset.

Han and colleagues described the presence of lung fibrotic-like abnormalities on follow-up CTs in 40 out of 114 patients $(35 \%)$ at 6 months after being discharged for COVID-19 pneumonia, and a reduction in DLCO in 27 out of 104 patients (26\%), the latter being more frequent in the group of patients with fibrotic-like changes on CT scan [6]. However, the authors list the presence of comorbidities, in particular chronic lung disease pre-existing the infection, as predisposing conditions for the development of fibrotic-like anomalies, which could at least partially explain the reduction in DLCO described in this group; furthermore, it is not clear whether the respiratory function tests of these patients remained stable compared to pre-COVID-19 pneumonia controls (as in our case series) or whether they deteriorated further. Similarly, the authors report that 37 patients had an intermediate CT follow-up at 3 months after the baseline CT, which already showed changes of fibrotic abnormalities, but they do not specify whether there was stability, improvement (as in our case), or worsening of the CT findings between this intermediate follow-up and the 6-month follow-up.

Similarly, we found signs of pulmonary architectural distortion in the 1st CT follow-up in 9 out of 22 patients (40\%) and analyzed in detail the evolution of fibrotic findings in the following months. Unexpectedly, in the 2nd follow-up, we found an almost complete regression of previously described lung lesions, with complete resorption of reticulations, parenchymal bands, and bronchial distortion, conditions that we had therefore misinterpreted as pulmonary fibrosis and "traction" bronchiectasis, both of which are irreversible. Patients who underwent a 3rd follow-up showed further improvement, with complete resolution of the alterations detected before.

We also evaluated the perilobular pattern, and the anatomical correspondence of the findings between the baseline $\mathrm{CT}$ and the 1st follow-up. These two elements could be relevant to the correct interpretation of findings seen in the remission phase of COVID-19 pneumonia. Specifically, we found the perilobular pattern, a well-known sign of organizing pneumonia (OP), in 2 out of 8 patients in the baseline CT and in 5 out of 8 in the 1st follow-up, and found interstitial changes (with fibrotic-like appearance) in parenchymal areas different to those affected at the baseline CT in 2 out of 8 patients, in one case with complete resorption of the lesions present in the baseline examination, showing a "migratory" behavior, another well-known sign of OP.

Therefore, our hypothesis is that parenchymal interstitial anomalies that can be seen in the short-term follow-up (1-2 months), such as parenchymal bands and reticulations, should not be immediately attributed to pulmonary fibrosis since they could rather be secondary to OP, whose lesions are slowly or very slowly resolving but reversible. Also, in this scenario, bronchiectasis should not be interpreted as "traction" bronchiectasis, which is irreversible, but rather as reversible bronchial distortion-ectasia, probably due to the traction exerted by the collapsed alveoli adjacent to the bronchi, which resolves consensually to the alveolar opacities. These findings are in agreement with other studies on recovering COVID-19 patients that describe an almost complete resolution of consolidations and bronchial dilatations after a period of 3-6 months and 1-year after discharge [10, 11, 21]. In particular, Zhao and colleagues recently analyzed follow-up CTs of 90 patients 1 year after discharge assessing the presence of several pulmonary CT features (i.e., GG opacities, subpleural lines, linear opacities, interlobular septal thickening, and reticulations), and 
found the presence of parenchymal abnormalities in 67 patients $(71 \%)$ but signs suggestive of pulmonary fibrosis in only 8 patients $(8.51 \%)$; the authors also report that patients with severe forms of pneumonia had a higher incidence of parenchymal changes at 1 year (including nonfibrotic changes) than those with mild forms and conclude that chest-CT imaging abnormalities caused by SARSCoV-2 could gradually resolve over time [11].

This hypothesis could have important implications in the management of these patients, particularly those who required prolonged hospitalization, in which an appropriate post-discharge steroid therapy could speed up the full recovery. This hypothesis would be consistent with the results of some case reports on the follow-up of COVID19 patients including histological data, describing a good response to steroid therapy in patients who developed OP after diffuse lung damage secondary to COVID-19 pneumonia [22].

The limitations of our study included its retrospective nature and the limited number of patients included. Another limitation was the lack of histopathological data to confirm our hypothesis. Therefore, larger and prospective multicenter studies are needed to confirm these hypotheses.

This case series, albeit of a small number of patients, suggests that pulmonary fibrosis-like abnormalities found after COVID-19 pneumonia could be, at least in some cases, transient and slowly resolving but still reversible, even in patients who required mechanical ventilation and prolonged hospitalization. Therefore, it could be reasonable to adopt a "wait and see" approach, with CT follow-up and the control of respiratory function, for a period of at least 9-12 months before considering invasive procedures like transbronchial biopsy or the administration of specific anti-fibrotic drugs, since both options come with risks and side effects.

Author Contribution A.P.: conceptualization, methodology, data curation, writing - original draft.

E.C, L.L.: resources, investigation, writing - reviewing and editing.

C.P., G.M., S.S., M.M.: resources, investigation.

M.Z: conceptualization, supervision, validation.

R.F.: supervision, validation.

As corresponding author, I confirm that the manuscript has been read and approved for submission by all the named authors.

Availability of Data and Material The datasets analyzed during the current study are available from the corresponding author on reasonable request.

Code Availability Not applicable.

\section{Declarations}

Ethics Approval The study was approved by the "Area Vasta Emilia Centro" Independent Ethical Committee (CE-AVEC; study ID:
EM335-2021-20111-EM1-OSS-AUSLIM) and was conducted in compliance with the Declaration of Helsinki.

Consent to Participate/for Publication Written informed consent was obtained from all patients included in the series.

All images were acquired as a standard of care.

Conflict of Interest The authors declare no competing interests.

\section{References}

1. WHO coronavirus disease (COVID-19) dashboard. Geneva: World Health Organization, 2020. Available online: https://covid 19.who.int/. Last cited: 03/11/2021.

2. Spagnolo P, Balestro E, Aliberti S, Cocconcelli E, Biondini D, Casa GD, Sverzellati N, Maher TM. Pulmonary fibrosis secondary to COVID-19: a call to arms? Lancet Respir Med. 2020;8(8):7502. https://doi.org/10.1016/S2213-2600(20)30222-8.

3. George PM, Wells AU, Jenkins RG. Pulmonary fibrosis and COVID-19: the potential role for antifibrotic therapy. Lancet Respir Med. 2020;8(8):807-15. https://doi.org/10.1016/S22132600(20)30225-3.

4. Gentile F, et al. COVID-19 and risk of pulmonary fibrosis: the importance of planning ahead. Eur J Prev Cardiol. 2020;27(13):1442-6. https://doi.org/10.1177/2047487320932695.

5. Raghu G, Wilson KC. COVID-19 interstitial pneumonia: monitoring the clinical course in survivors. Lancet Respir Med. 2020;8(9):839-42. https://doi.org/10.1016/S2213-2600(20) 30349-0.

6. Han X, Fan Y, Alwalid O, Li N, Jia X, Yuan M, Li Y, Cao Y, Gu J, Wu H, Shi H. Six-month follow-up chest CT findings after severe COVID-19 pneumonia. Radiology. 2021;299(1):E177-86. https:// doi.org/10.1148/radiol.2021203153.

7. Yu M, Liu Y, Xu D, Zhang R, Lan L, Xu H. Prediction of the development of pulmonary fibrosis using serial thin-section CT and clinical features in patients discharged after treatment for COVID-19 pneumonia. Korean J Radiol. 2020;21(6):746-55. https://doi.org/10.3348/kjr.2020.0215.

8. Wei J, Yang H, Lei P, Fan B, Qiu Y, Zeng B, Yu P, Lv J, Jian Y, Wan CJ. Analysis of thin-section CT in patients with coronavirus disease (COVID-19) after hospital discharge. Xray Sci Technol. 2020;28(3):383-9. https://doi.org/10.3233/XST-200685.

9. Shah AS, Wong AW, Hague CJ, Murphy DT, Johnston JC, Ryerson CJ, Carlsten C. A prospective study of 12-week respiratory outcomes in COVID-19-related hospitalisations. Thorax. 2020;76(4):402-4. https://doi.org/10.1136/thora xjnl-2020-216308.

10. Lerum TV, Aaløkken TM, Brønstad E, Aarli B, Ikdahl E, Lund KMA, et al. Dyspnoea, lung function and CT findings three months after hospital admission for COVID-19. Eur Respir J. 2020;2003448. https://doi.org/10.1183/13993003.03448-2020.

11. Zhao Y, Yang C, An X, Xiong Y, Shang Y, He J, et al. Follow-up study on the COVID-19 survivors after one year discharged from hospital. Int J Infect Dis. 2021;S1201-9712(21)00726-8. https:// doi.org/10.1016/j.ijid.2021.09.017.

12. Hansell DM, Bankier AA, MacMahon H, McLoud TC, Müller NL, Remy J. Fleischner Society: glossary of terms for thoracic imaging. Radiology. 2008;246(3):697-722. https://doi.org/10. 1148/radiol.2462070712.

13. Simpson S, Kay FU, Abbara S, Bhalla S, Chung JH, Chung M, Henry TS, Kanne JP, Kligerman S, Ko JP, Litt H. Radiological Society of North America expert consensus statement on reporting chest CT findings related to COVID-19. Endorsed by the 
Society of Thoracic Radiology, the American College of Radiology, and RSNA - Secondary Publication. J Thorac Imaging. 2020;35(4):219-27. https://doi.org/10.1097/RTI.0000000000 000524.

14. Kwee TC, Kwee RM. Chest CT in COVID-19: what the radiologist needs to know. Radiographics. 2020;40(7):1848-65. https:// doi.org/10.1148/rg.2020200159.

15. Best AC, Meng J, Lynch AM, Bozic CM, Miller D, Grunwald GK, Lynch DA. Idiopathic pulmonary fibrosis: physiologic tests, quantitative CT indexes, and CT visual scores as predictors of mortality. Radiology. 2008;246(3):935-40. https://doi.org/10. 1148/radiol.2463062200.

16 Sverzellati N, Calabrò E, Chetta A, Concari G, Larici AR, Mereu M, Cobelli R, De Filippo M, Zompatori M. Visual score and quantitative CT indices in pulmonary fibrosis: relationship with physiologic impairment. Radiol Med. 2007;112(8):1160-72. https:// doi.org/10.1007/s11547-007-0213-x. English, Italian.

17. Antonio GE, Wong KT, Hui DS, Wu A, Lee N, Yuen EH, Leung CB, Rainer TH, Cameron P, Chung SS, Sung JJ, Ahuja AT. Thinsection $\mathrm{CT}$ in patients with severe acute respiratory syndrome following hospital discharge: preliminary experience. Radiology. 2003;228(3):810-5. https://doi.org/10.1148/radiol.2283030726.

18. Wong KT, Antonio GE, Hui DS, Ho C, Chan PN, Ng WH, Shing $\mathrm{KK}, \mathrm{Wu} \mathrm{A}$, Lee N, et al. Severe acute respiratory syndrome: thin-section computed tomography features, temporal changes, and clinicoradiologic correlation during the convalescent period. J Comput Assist Tomogr. 2004;28(6):790-5. https://doi.org/10. 1097/00004728-200411000-00010.

19. Ujita M, Renzoni EA, Veeraraghavan S, Wells AU, Hansell DM. Organizing pneumonia: perilobular pattern at thin-section CT. Radiology. 2004;232(3):757-61. https://doi.org/10.1148/radiol. 2323031059.

20. Wu J, Pan J, Teng D, Xu X, Feng J, Chen YC. Interpretation of CT signs of 2019 novel coronavirus (COVID-19) pneumonia. Eur Radiol. 2020;30(10):5455-62. https://doi.org/10.1007/ s00330-020-06915-5.

21. Sonnweber T, Sahanic S, Pizzini A, Luger A, Schwabl C, Sonnweber B, et al. Cardiopulmonary recovery after COVID-19 - an observational prospective multi-center trial. Eur Respir J. 2020;2003481. https://doi.org/10.1183/13993003.03481-2020.

22. Vadász I, Husain-Syed F, Dorfmüller P, Roller FC, Tello K, Hecker M, et al. Severe organizing pneumonia following COVID19. Thorax. 2020;thoraxjnl-2020-216088. https://doi.org/10.1136/ thoraxjnl-2020-216088.

Publisher's Note Springer Nature remains neutral with regard to jurisdictional claims in published maps and institutional affiliations. 